\title{
Crossmodal transfer of form discrimination in preschool children'
}

MARION BLANK, L. DORIS ALTMAN, AND WAGNER H. BRIDGER ALBERT EINSTEIN COLLEGE OF MEDICINE ${ }^{2}$

Children, three to four years of age, were given crossmodal transfer problems involving presentation of identical form discriminations in the visual and tactual modalities. Transfer, without relevant verbalization, was obtained from vision to touch, but not from touch to vision. This transfer suggests that human beings have nonverbal representation available for problem solving.

A long history of controversy exists concerning whether man is qualitatively different from other animals. Despite our speculations, it has been extremely difficult to find experimental evidence of basic nonverbal skills in which man has unique learning abilities. The phenomenon of crossmodal transfer stands as an exception to this generalization since it has been found that subhuman primates are very weak in this behavior (Moffett \& Ettlinger, 1966; Wegener, 1965; Wilson \& Shaffer, 1963), e.g., in using information gained visually to help them solve a tactual problem.

It has been suggested that the superiority of humans on this task is due to their verbal abilities (Ettlinger, 1967). Our previous research (Blank \& Bridger, 1964) has led us to believe, however, that not all levels of crossmodal transfer need be language dependent. Language was required when the stimuli across modalities were so physically dissimilar that only a concept could unify them (e.g., "two lights" in a first problem could be seen as similar to "two sounds" in a second problem if the concept of number was used). However, our findings suggested that transfer involving the identical stimulus experienced in two modalities need not be language dependent (e.g., experiencing a ball tactually in the first problem and then the same ball visually in the second problem).

If this latter type of transfer occurs without language, it represents an important cognitive achievement. Transfer across modalities means that the organism must have retained some inner nonverbal representation of the object since the sensory input on the transfer problem (e.g., a tactual stimulus) is totally different from the sensory input on the training problem (e.g., a visual stimulus).

The design of the experiment was as follows: Preschool age children were trained on a discrimination problem in one sensory modality, either vision or touch, and were then transferred to the same problem in the other modality. The transfer problem required the selection of either the previously correct form in the other modality (identical transfer) or the previously incorrect form (reversal transfer).
The reversal technique was used as our control since previous research (Moffett \& Ettlinger, 1966) indicated that it: (1) provides equivalent training for all groups and thereby controls for nonspecific transfer such as warm-up; (2) accentuates any difference in specific transfer that may exist between the identical and reversal transfer groups (i.e., if the child is guided by having been rewarded for a consistent object during training, he should show positive transfer when rewarded on the same object on the transfer problem and negative transfer if rewarded on the opposite object).

\section{Subjects}

The Ss were 54 children, 26 males and 28 females, who ranged in age from 41-59 months, with a mean age of 50.8 months. Because transfer cannot be evaluated unless $S$ passes the training problem, Ss who failed that problem were excluded from the study. The Ss were tested until there were 10 in each of four groups: two visual training groups, one given identical tactual transfer and the other reversal tactual transfer, and two tactual training groups, one given identical visual transfer and the other reversal visual transfer. Procedure

Each $\mathrm{S}$ was tested individually in a small quiet room in the day care center. Prior to the experimental tasks, the children were given eight pretraining problems in making crossmodal equivalence judgments (tactual and visual) to aid them in focusing their attention on the dimension of form. As described in our previous research (Blank \& Bridger, 1964) these problems involved selecting visually which one of two forms was identical to a tactually perceived form.

The experimental tasks were composed of a training and a test problem; each consisted of a two-choice simultaneous discrimination of three-dimensional geometric forms. The discriminanda were an inverted bridge-shaped figure and a rectangle, neither of which was used in the pretraining. Half the Ss were reinforced for one form, half for the other. The discriminanda, whose positions were randomly changed across trials, were located over wells which could contain a small candy reward. The $\mathrm{S}$ was told that the candy would always be under one of the toys and never under the other.

For the visual problem the objects, enclosed in clear plastic boxes, were presented on a platform directly in front of $S$. A curtain was drawn between $S$ and $E$ after each trial to prevent the $S$ from seeing any manipulation of material. For the tactual problem an enclosed box $12 \times 8 \times 7$ in. was used. It had two 
Table 1

Crossmodal Transfer of Form Discrimination

\begin{tabular}{lccccc} 
& \multicolumn{2}{c}{ Visual to Tactual } & \multicolumn{3}{c}{ Tactual to Visual } \\
\hline & Identical & Reversal & & Identical & Reversal \\
Training M & 12.1 & 12.9 & M & 3.4 & 5.0 \\
Problem SD & 9.2 & 13.0 & SD & 4.4 & 6.7 \\
& & & & & \\
Test M & 0.7 & 3.9 & M & 12.6 & 16.5 \\
Problem SD & 0.7 & 3.8 & SD & 13.8 & 14.1 \\
\hline
\end{tabular}

openings through which $\mathbf{S}$ could insert his hands without being able to see the discriminanda.

The criterion of learning on both training and test problems was nine correct responses in 10 successive trials. A maximum of 40 trials was given on any day with testing continued over two days when necessary. When criterion was reached on the training problem, the transfer problem was immediately introduced at that session. A maximum of 60 and 30 trials, respectively, was given on the training and test problems.

The identical transfer group was rewarded on the same form that was previously correct, the reversal transfer group was rewarded on the previously incorrect form. The score of 30 attained by Ss who failed the test problem was included in the data analysis since failure on the test problem is a major index of whether transfer has occurred. A maximum of 30 trials was given on the transfer problem because its continuation on the following day would have been tantamount to presenting a new problem.

\section{Results and Discussion}

Table 1 presents results of the scores on the training and test problems in each sensory modality. Because there was such a large difference between visual and tactual training problems (Mann-Whitney $U=104, \mathrm{Ns}=20, \mathrm{p}<.02$ ), the test problems in the different modalities were analyzed separately.

Analysis of the difference between the identical and reversal transfer groups was not significant in the visual modality $(U=32.5, \mathrm{Ns}=10, \mathrm{p}>.05)$ but was significant in the tactual modality $(U=19, \mathrm{Ns}=10, \mathrm{p}<.01)$. Although no significant transfer occurred from the tactual to the visual modality, the results do indicate transfer from the visual to the tactual modality. This transfer seems even more striking when one considers that four of the 10 children in the "identical" group immediately selected the correct stimulus on the tactual transfer problem.

Despite the fact that transfer was in one direction, these findings nevertheless indicate that crossmodal transfer of form discrimination can occur in young children. In addition, this transfer does not seem to be mediated by language. The shapes used could not be readily labeled by young children and even with posttest questioning not one of the children could offer any labels for the stimull. This finding is in marked contrast to our previous research (Blank \& Bridger, 1964) on crossmodal concepts (one vs two lights, one vs two sounds) in which language was used by all children who achieved crossmodal transfer. These findings also indicate that there may not be a distinction, as Ettlinger suggested, between the need for language in transfer, but not in matching. Language is needed when an abstract concept such as number is required in the crossmodal problem and not in crossmodal transfer per se. Transfer involving the identical forms does not appear to require such abstract conceptualization and thus can be achieved without language.

The fact that this transfer was achieved without language suggests that young children have nonverbal means of representation. Although it was not possible to determine what mechanism mediated this transfer, it seems likely that imagery may have been used; that is, when the tactual stimulus was presented, the child evoked a visual image which corresponded to the previously experienced visual stimulus. These results suggest that, even if animals have images, their great difficulty in achieving crossmodal transfer may mean that they are unable to readily evoke these images in relevant situations.

The question remains as to why transfer did not occur when the training task was tactual and the test problem visual. Two possibilities seem most likely: One is that the visual modality is prepotent so that its stimulus impact suppresses the influence of information gained in any other modality. A second possibility is that information gained through the tactual modality is not well coded or internalized (i.e., an image is not easily evoked). As a result tactual information may not be retained in a form that can be readily used in crossmodal problem solving. It should be noted that these two possibilities may be complementary rather than contradictory.

\section{References}

BLANK, M., \& BRIDGER, W. H. Crossmodal transfer in nursery school children. J. comp. Physiol Psychol, 1964, 58, 277.

ETTLINGER, G. Analysis of cross-modal effects and their relationship to language. Brain mechanisms underlying speech and language. New York: Grune \& Stratton, 1967, 53-60.

MOFFETT, A., \& ETTLINGER, G. Opposite responding in two sense modalities. Science, 1966, 153, 205.

WEGENER, J. G. Cross-modal transfer in monkeys. J. comp. Physiol. Psychol., 1965, 59, 450.

WILSON, W. A., JR., \& SHAFFER, O. C. Intermodality transfer of specific discriminations in the monkey. Nature, 1963, 197, 107. Notes

1. This research was supported by USPHS Grants No. K3 MH 10, 749, $\mathrm{K} 3 \mathrm{MH} \mathrm{4177}$, and $\mathrm{MH} 08498$. The authors express their appreciation to the Rochambeau School, White Plains and the Victory and Bronxdale Day Care Centers, Bronx, N. Y. for their cooperation.

2. Department of Psychiatry, Albert Einstein College of Medicine, Yeshiva University, Bronx, New York. 